\title{
Light Hadron Spectroscopy at BEPC
}

\author{
Bing-Song Zou ${ }^{\mathrm{a}}$
}

${ }^{a}$ CCAST (World Laboratory), P.O. Box 8730, Beijing 100080 and Institute of High

Energy Physics, CAS, P. O. Box 918(4), Beijing 100039, P.R.China

The $J / \Psi$ and $\Psi^{\prime}$ experiments at the Beijing Electron Positron Collider (BEPC) play a unique role in many aspects of light hadron spectroscopy, such as hunting for glueballs and hybrids, extracting $u \bar{u}+d \bar{d}$ and $s \bar{s}$ components of mesons, and studying excited nucleons and hyperons, i.e., $N^{*}, \Lambda^{*}, \Sigma^{*}$ and $\Xi^{*}$ resonances. Physics objectives, recent results and future prospects of light hadron spectroscopy at BEPC are presented.

\section{Introduction}

There are two kinds of hadrons: mesons and baryons. They are the smallest particles with sub-structure observed. Although we have already known for more than twenty years that hadrons are composed of quarks and gluons governed by strong interaction QCD, we still do not know how they are built up from these partons. The purpose of hadron spectroscopy is to explore the internal quark-gluon structure of the hadrons and the underlying strong interaction QCD. It is a fundamental task for physicists.

The Institute of High Energy Physics at Beijing runs an electron-positron collider (BEPC) with a general purpose solenoidal detector, the BEijing Spectrometer (BES) 迎, which is designed to study exclusive final states in $e^{+} e^{-}$annihilations at the center of mass energy from 2000 to $5600 \mathrm{MeV}$. In this energy range, the largest cross sections are at the $J / \Psi(3097)$ and $\Psi^{\prime}(3686)$ resonant peaks. At present, the BES has collected about 30 million $J / \Psi$ events and 3.7 million $\Psi^{\prime}$ events. More data are going to be taken. From $J / \Psi$ and $\Psi^{\prime}$ decays, both meson spectroscopy and baryon spectroscopy can be studied.

Three main processes which play a unique role for the light hadron spectroscopy are $\Psi$ radiative decay, $\Psi$ hadronic decay into mesons, and $\Psi$ hadronic decay into baryons and anti-baryons. In the following three sections, I will outline the physics objectives and summarize recent results for each of them. The outlook is given in the final section.

\section{2. $\Psi$ radiative decays}

There are three main physics objectives for $\Psi$ radiative decays:

(1) Looking for glueballs and hybrids. As shown in Fig. 1, after emitting a photon, the $c \bar{c}$ pair is in a $C=+1$ state and decays to hadrons dominantly through two gluon intermediate states. Simply counting the power of $\alpha_{s}$ we know that glueballs should have the largest production rate, hybrids the second, then the ordinary $q \bar{q}$ mesons. 


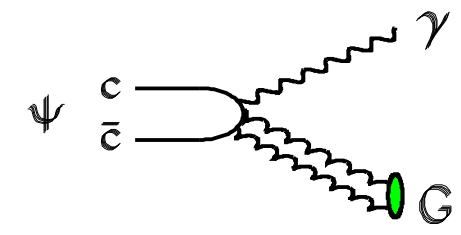

(a)

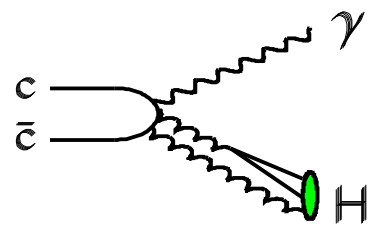

(b)

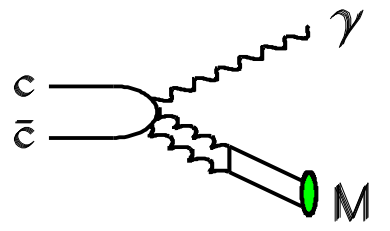

(c)

Figure 1. $\Psi$ radiative decays to (a) glueball, (b) hybrid, and (c) $q \bar{q}$ meson.

(2) Completing $q \bar{q}$ meson spectroscopy and studying their production and decay rates, which is crucial for understanding their internal structure and confinement.

(3) Extracting $g g \leftrightarrow q \bar{q}$ coupling from perturbative energy region of above $3 \mathrm{GeV}$ to nonperturbative region of $0.3 \mathrm{GeV}$. This may show us some phenomenological pattern for the smooth transition from perturbative QCD to strong nonperturbative QCD.

Up to now, we have mainly worked on glueball searches. One thing worth noting is that the $J / \Psi$ radiative decay has a similar decay pattern as $0^{-+}, 0^{++}$and $2^{++}$charmoniums, i.e., $\eta_{c}, \chi_{c 0}$ and $\chi_{c 2}$, as it should be, since all of them decay through two gluons. The $4 \pi, \bar{K} K \pi \pi, \eta \pi \pi$ and $\bar{K} K \pi$ seem to be the most favorable final states for the two gluon transition. The branching ratios for $J / \Psi$ radiative decay to these four channels are listed in Table 1. The sum of them is about half of all radiative decays. If glueballs exist, they should appear in these four channels. Therefore BES Collaboration has recently performed partial wave analyses (PWA) of these four channels [3 [6] as well as $\gamma \bar{K} K$ channel[7],8]. The hadronic invariant mass spectra for these channels are shown in Figs. 2-6.

Table 1

Branching ratios for the four largest $J / \Psi$ radiative decay channels $\left(\mathrm{BR} \times 10^{3}\right)$

\begin{tabular}{cccc}
\hline$\gamma 4 \pi$ & $\gamma \bar{K} K \pi \pi$ & $\gamma \eta \pi \pi$ & $\gamma \bar{K} K \pi$ \\
\hline $14.4 \pm 1.8$ [2] & $9.5 \pm 2.7$ 田] & $6.1 \pm 1.0$ [9] & $6.0 \pm 2.1$ [6] \\
\hline
\end{tabular}

The BES results on $J / \Psi \rightarrow \gamma \pi^{+} \pi^{-} \pi^{+} \pi^{-}$[3] are approximately consistent with the results from the earlier re-analysis of MARKIII data[10] where three $0^{++}$resonances are needed at $(1500,1750,2100) \mathrm{MeV}$ decaying dominantly through $\sigma \sigma$ intermediate state, plus a very broad $0^{-+}$component named $\eta(1800)$ first [5] and $\eta(2190)$ later [11]. But the BES data favor more $2^{++}$in the high mass region. A broad $2^{++}$resonance $f_{2}(1950)$ is needed around $2 \mathrm{GeV}$ together with some contribution from $f_{2}(1270)$ and $f_{2}(1565)$.

For $J / \Psi \rightarrow \gamma K^{+} K^{-} \pi^{+} \pi^{-}\left[4\right.$, we found that the $K^{*} \bar{K}^{*}$ contribution peaks strongly near threshold, which can be fitted with a broad $0^{-+}$resonance compatible with $\eta(2190)$ [11]. The data also definitely required a broad $2^{++}$resonance $f_{2}(1950)$ decaying to $K^{*} \bar{K}^{*}$. There is further evidence for a $2^{-+}$component peaking at $2.55 \mathrm{GeV}$. The non- $K^{*} \bar{K}^{*}$ 

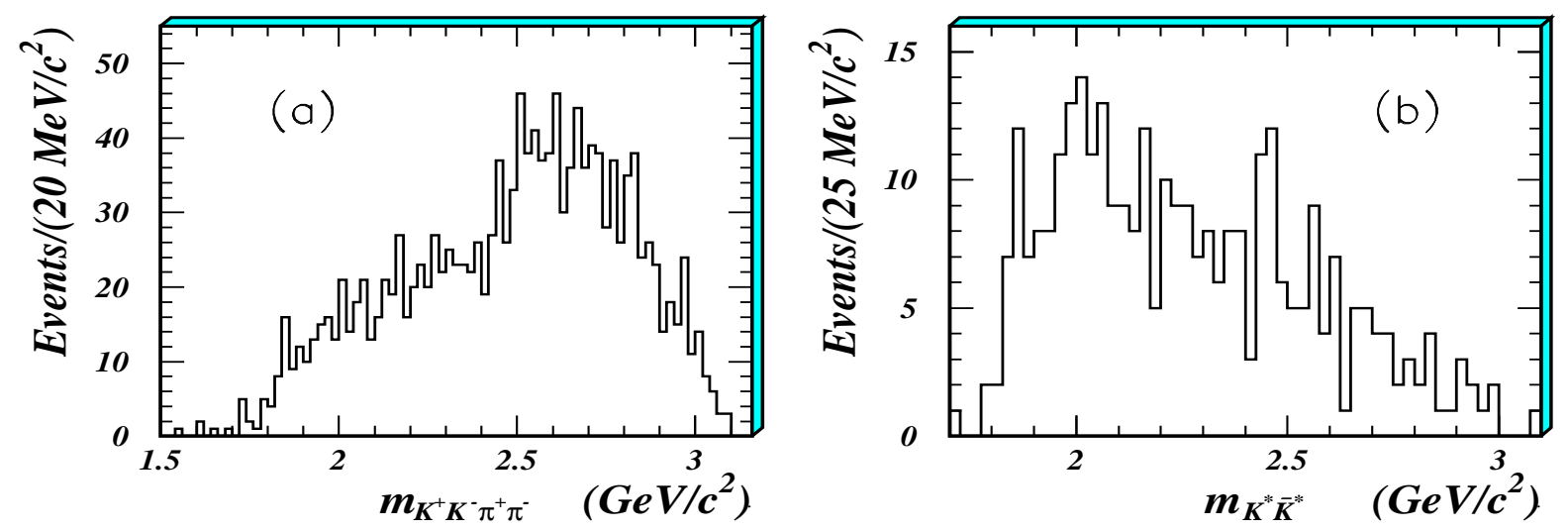

Figure 2. (a) The $K K \pi \pi$ mass of $J / \Psi \rightarrow \gamma K^{+} K^{-} \pi^{+} \pi^{-}$; (b) The $K^{*} \bar{K}^{*}$ mass of $J / \Psi \rightarrow$ $\gamma K^{*} \bar{K}^{*}$. [4]

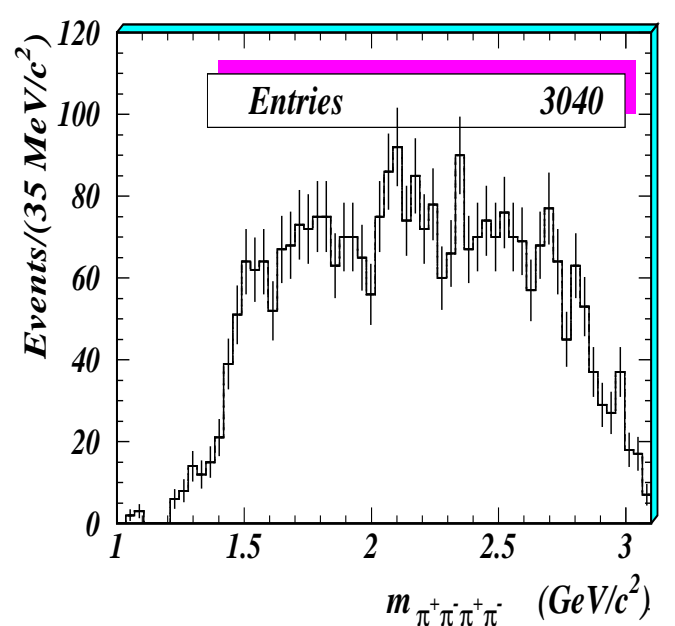

Figure 3. The $4 \pi$ mass of $J / \Psi \rightarrow$ $\gamma \pi^{+} \pi^{-} \pi^{+} \pi^{-} \cdot 3$

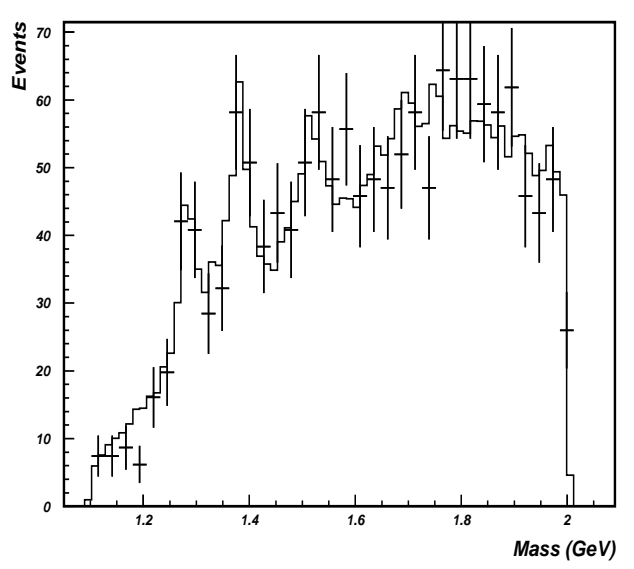

Figure 5. The $\eta \pi \pi$ mass of $J / \Psi \rightarrow$ $\gamma \eta \pi^{+} \pi^{-} \cdot[5$

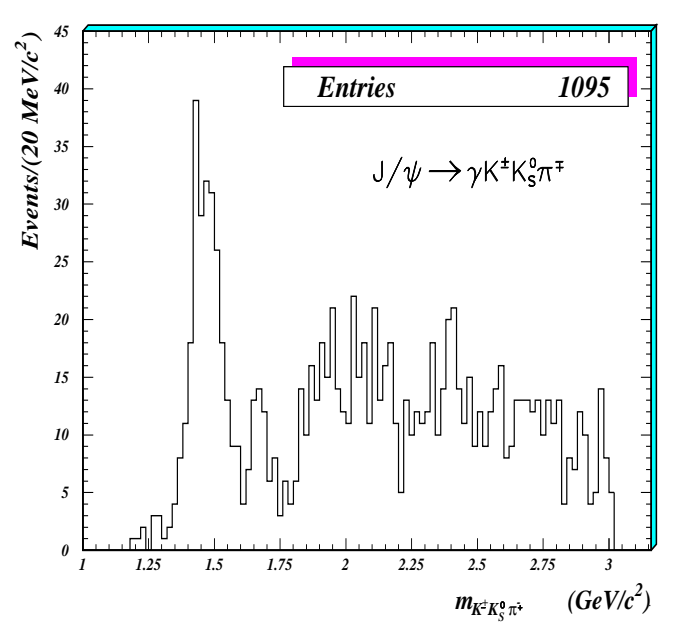

Figure 4. The $K_{S} K^{ \pm} \pi^{\mp}$ mass of $J / \Psi \rightarrow \gamma K_{S} K^{ \pm} \pi^{\mp} \cdot[6]$

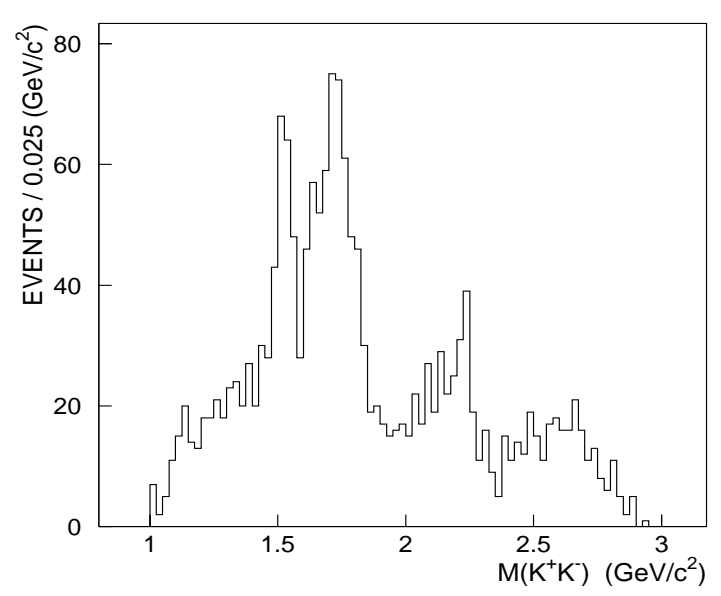

Figure 6. The $K \bar{K}$ mass of $J / \Psi \rightarrow$ $\gamma K^{+} K^{-} \cdot[7]$ 
contribution is close to phase space; it peaks at $2.6 \mathrm{GeV}$ and is very different from $K^{*} \bar{K}^{*}$.

For $J / \Psi \rightarrow \gamma \eta \pi^{+} \pi^{-}[5]$, the main discovery from the BES data is that the $0^{-+}$contribution dominates with three $0^{-+}$resonances, $\eta(1440), \eta(1760)$ and $\eta(2190)$ (named $\eta(1800)$ at that time). In addition, there is a definite $2^{-+}$signal for the $\eta_{2}(1870)$ decaying dominantly to $f_{2} \sigma$.

The structures in $J / \Psi \rightarrow \gamma K^{+} K^{-} \pi^{0}$ and $K_{S} K^{ \pm} \pi^{\mp}$ [6] are relatively simple. Two largest components are from $0^{-+}$resonances $\eta(1440)$ and $\eta(2040)$.

We also re-analyzed the $J / \Psi \rightarrow \gamma K^{+} K^{-}$data, $0^{++}$contribution $(75 \% \sim 80 \%)$ dominates the $f_{J}(1710)$ peak [7, 8]. Previous moment analysis suffers a problem of incomplete detection phase space 12 .

From these analyses together with information from other sources [9, 13], we get $J / \Psi$ radiative decay branching ratios for the production of $0^{-+}, 2^{++}, 0^{++}$and $2^{-+}$resonances of $1 \sim 2.5 \mathrm{GeV}$ as listed in Table2.

Table 2

$J / \Psi$ radiative decay branching ratios for the production of $0^{-+}, 2^{++}, 0^{++}$and $2^{-+}$resonances of $1 \sim 2.5 \mathrm{GeV}\left(\mathrm{BR} \times 10^{3}\right)$

\begin{tabular}{|c|c|c|c|c|c|}
\hline$\eta(2190)$ & $19 \pm 3$ [14, 3 & $f_{2}(1950)$ & $2.6 \pm 0.6[14,3,4]$ & $f_{0}(1500)$ & $1.1 \pm 0.1$ [3,9] \\
\hline$\eta(1440)$ & $2.1 \pm 0.7$ [5,6] & $f_{2}(1270)$ & $1.4 \pm 0.19$ & $f_{0}(1740)$ & $1.1 \pm 0.1$ [3,7] \\
\hline$\eta(2040)$ & $2.1 \pm 0.76$ & $f_{2}(15$ & \pm 0.1 [3] & & $0.7 \pm 0.2$ \\
\hline$\eta(1760)$ & $1.8 \pm 0.8$ & $f_{2}^{\prime}(1525)$ & $0.5 \pm 0.19$ & $\eta_{2}(1870)$ & $0.9 \pm 0.3$ \\
\hline
\end{tabular}

The largest branching ratio of the $J / \Psi$ radiative decay is for the very broad $0^{-+}$resonance $\eta(2190)$ with a full width of $(850 \pm 100) \mathrm{MeV}$. It decays to $\rho \rho, \omega \omega, K^{*} \bar{K}^{*}$ and $\phi \phi$ in a flavor-blind way within errors [11]. These properties suggest that it is the $0^{-}$glueball predicted near this mass by lattice calculations. Its mixing with other $0^{-+}$resonances will give a natural explanation for the large production rates for all the $0^{-+}$resonances listed in the Table2, which has long been known as the pseudoscalar puzzle 2].

The largest $2^{++}$production rate is for the broad $f_{2}(1950)$ decaying to $4 \pi[3]$ and $K^{*} \bar{K}^{*}[$ [䧃]. It also appears in two other glue-rich processes: proton-proton central production [15] and proton-antiproton annihilation 16. Especially, it has a much larger production rate 15 in the central production process than other nearby $2^{++}$resonances above $1.5 \mathrm{GeV}$. These properties make the $f_{2}(1950)$ the best $2^{++}$glueball candidate 14.

The two $0^{++}$resonances, $f_{0}(1500)$ and $f_{0}(1740 \pm 30)$, have similar production rates in the $J / \Psi$ radiative decays. However, the $f_{0}(1740 \pm 30)$ has much weaker production rates than the $f_{0}(1500)$ in two other glue-rich processes [15, 16] and much stronger production rates in glueball disfavoring processes such as the photon-photon collision [17] and the $J / \Psi \rightarrow \omega K^{+} K^{-}$process [18]. This makes the $f_{0}(1500)$ the best $0^{++}$glueball candidate. But it is possible that the bare glueball state is dispersed over three real resonances, $f_{0}(1500), f_{0}(1740)$ and the nearby broad state $f_{0}(1670)$ [19] or $f_{0}(1530)$ [20] or $f_{0}(1370)$ [21].

In summary, from our partial wave analyses of the four largest $J / \Psi$ radiative decays together with information from other sources, we find that $f_{0}(1500), \eta(2190)$ and $f_{2}(1950)$ 
display the strongest glueball characteristics. Their mass ratios agree well with the latest predictions of Lattice Gauge calculations of the glueball spectrum [14].

As for the isoscalar $1^{-+}$hybrids, they are predicted to decay dominantly into $4 \pi$ through $a_{1} \pi$ and $\pi(1300) \pi$ intermediate states [22]. If they exist, they should appear in the $J / \Psi \rightarrow$ $\gamma \pi^{+} \pi^{-} \pi^{+} \pi^{-}$process. In our present partial wave analysis of this channel, we have not include this exotic quantum number. We shall re-analyze this channel by allowing this possibility after more statistics on this channel is available.

A special subsector of $J / \Psi$ radiative decays, $J / \Psi \rightarrow \gamma A \rightarrow \gamma(\gamma \rho: \gamma \omega: \gamma \phi)$, is on our list for the partial wave analysis in near future. This process filters the flavor content of all $C=+$ states and disfavors the glueball production since glueballs do not couple to the photon directly. BES data for $J / \Psi \rightarrow \gamma(\gamma \rho: \gamma \phi)$ show clear evidence for the $\eta(1440)$ decaying to $\gamma \rho$ and $\gamma \phi$ [23.

\section{3. $\Psi$ hadronic decays to mesons}

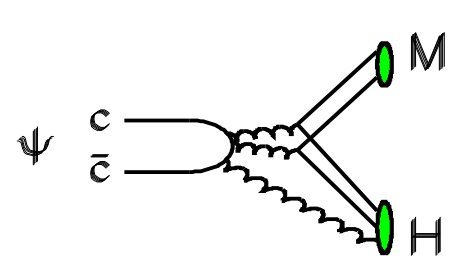

(a)

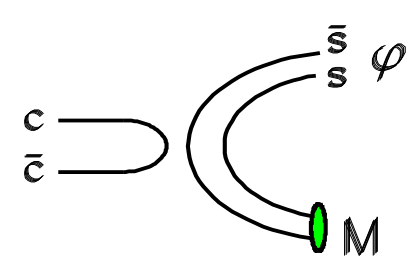

(b)

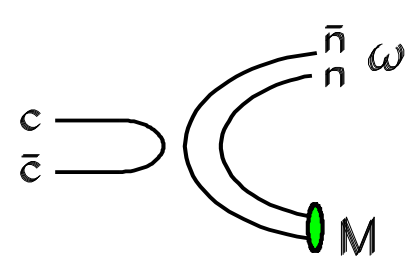

(c)

Figure 7. $\Psi$ hadronic decays to (a) hybrids, (b) $s \bar{s}$, and (c) $n \bar{n} \equiv \frac{1}{\sqrt{2}}(u \bar{u}+d \bar{d})$ mesons.

There are also mainly three physics objectives here:

(1) Looking for hybrids. Since $\Psi$ decays to hadrons through three gluons, final states involving a hybrid as shown in Fig. 1(a) are expected to have larger production rate than ordinary $q \bar{q}$ mesons as shown in Fig. $7(\mathrm{~b}, \mathrm{c})$.

(2) Extracting $u \bar{u}+d \bar{d}$ and $s \bar{s}$ components of associated mesons, M, via $\Psi \rightarrow M+\omega / \phi$ as shown in Fig. Ø (b,c).

(3) Disfavoring glueball production. We can analyze the quark/gluon content of a particle, M, by comparing its production in $\Psi \rightarrow \gamma M, \omega M$ and $\phi M$.

In order to look for isoscalar $1^{-+}$hybrid $\hat{\omega}$ decaying to $4 \pi$, we have studied $J / \Psi \rightarrow$ $\omega \pi^{+} \pi^{-} \pi^{+} \pi^{-}$process 24. A peak around $1.75 \mathrm{GeV}$ in the $4 \pi$ invariant mass spectrum is visible. But due to low statistics, no PWA is performed. No other structure is observed.

To investigate the $u \bar{u}+d \bar{d}$ and $s \bar{s}$ components of mesons, we have studied $J / \psi \rightarrow$ $\omega \pi^{+} \pi^{-}, \omega K^{+} K^{-}, \phi \pi^{+} \pi^{-}$and $\phi K^{+} K^{-}$channels. The invariant mass spectra for these channels are similar to the previous ones by MARKIII and DM2 Collaborations.

For $J / \psi \rightarrow \omega \pi^{+} \pi^{-}$, there are two clear peaks at $500 \mathrm{MeV}$ and $1275 \mathrm{MeV}$ in the $2 \pi$ mass spectrum corresponding to the $\sigma$ and the $f_{2}(1275)$, respectively [8]. For $J / \psi \rightarrow \omega K^{+} K^{-}$, 
there is a threshold enhancement due to the $f_{0}(980)$ and a clear peak at $1710 \mathrm{MeV}$ probably due to the $f_{0}(1710)$.

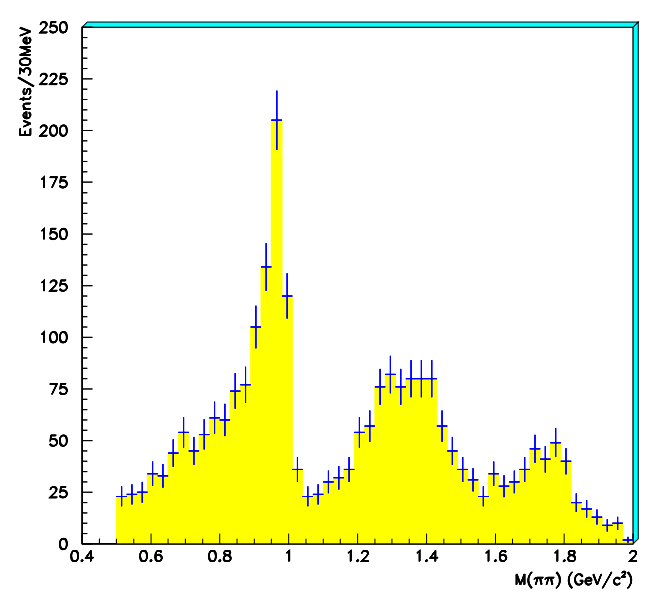

Figure 8. The $\pi \pi$ mass of $J / \Psi \rightarrow \phi \pi^{+} \pi^{-}$

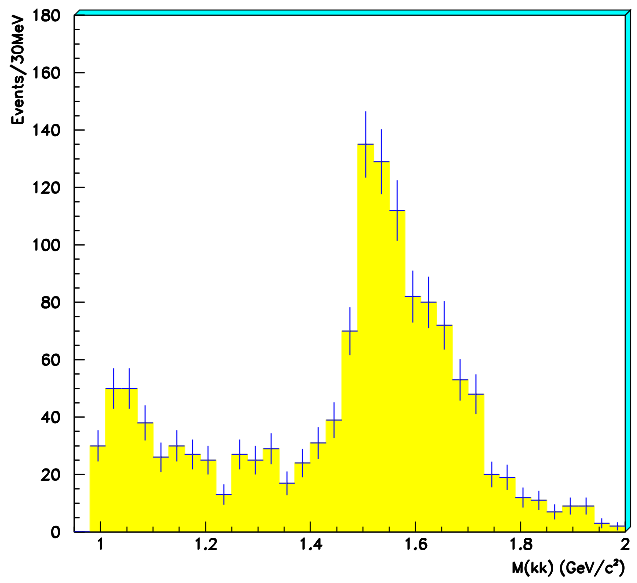

Figure 9. The $K \bar{K}$ mass of $J / \Psi \rightarrow$ $\phi K^{+} K^{-}$.

The $\pi \pi$ and $K \bar{K}$ invariant mass spectra for the $J / \Psi \rightarrow \phi \pi^{+} \pi^{-}$and $J / \Psi \rightarrow \phi K^{+} K^{-}$ based on the $2.3 \times 10^{7} \mathrm{~J} / \Psi$ events of BESII are shown in Fig. 8 and Fig. 9, respectively. Partial wave analyses are performed for these two channels. Preliminary results 8 indicate that (1) in the $\pi \pi$ mass spectrum of the $J / \Psi \rightarrow \phi \pi^{+} \pi^{-}$process all three peaks at $980 \mathrm{MeV}$, $1330 \mathrm{MeV}$ and $1770 \mathrm{MeV}$ are dominantly $0^{++}$; (2) in the $K \bar{K}$ mass spectrum of the $J / \Psi \rightarrow$ $\phi K^{+} K^{-}$the peak at $1525 \mathrm{MeV}$ is due to $f_{2}^{\prime}(1525)$ while the $K \bar{K}$ threshold enhancement and the shoulder around $1700 \mathrm{MeV}$ are due to $f_{0}(980)$ and $f_{0}(1710)$, respectively. The $f_{0}(1770)$ in the $J / \Psi \rightarrow \phi \pi^{+} \pi^{-}$and the $f_{0}(1710)$ in the $J / \Psi \rightarrow \phi K^{+} K^{-}$seem to be two separate resonances. The $f_{0}(1710 \pm 20) \rightarrow K \bar{K}$ appears clearly in the $J / \Psi \rightarrow$ $\gamma K^{+} K^{-}, \omega K^{+} K^{-}$and $\phi K^{+} K^{-}$; while the $f_{0}(1760 \pm 20)$ appears in $J / \Psi \rightarrow \gamma \pi^{+} \pi^{-} \pi^{+} \pi^{-}$, $\omega \pi^{+} \pi^{-} \pi^{+} \pi^{-}$and $\phi \pi^{+} \pi^{-}$.

In summary, the $f_{0}(1710-1770)$ appears clearly in many $J / \Psi \rightarrow \omega / \phi+X$ channels while $f_{0}(1500)$ is hardly visible in any of these glueball-disfavored processes. The $\sigma$ and $f_{2}(1275)$ appear clearly only in the $J / \Psi \rightarrow \omega+X$ process, the $f_{2}^{\prime}(1525)$ appears clearly only in the $J / \Psi \rightarrow \phi+X$ process, and $f_{0}(980)$ appears clearly in both processes.

\section{4. $\Psi$ hadronic decays to baryons and anti-baryons}

Baryons are the basic building blocks of our world. To understand the internal quarkgluon structure of baryons is one of the most important tasks in nowadays particle and nuclear physics. From theoretical point of view, since baryons represent the simplest system in which the three colors of QCD neutralize into colorless objects and the essential non-Abelian character of QCD is manifest, the systematic study of various baryon spectroscopy will provide us with critical insights into the nature of QCD in the confinement domain 25,26. 
$J / \Psi$ and $\Psi^{\prime}$ decays provide an excellent place for studying excited nucleons and hyperons $-N^{*}, \Lambda^{*}, \Sigma^{*}$ and $\Xi^{*}$ resonances. The corresponding Feynman graph for the production of these excited nucleons and hyperons is shown in Fig. 10 where $\Psi$ represents either $J / \Psi$ or $\Psi^{\prime}$.

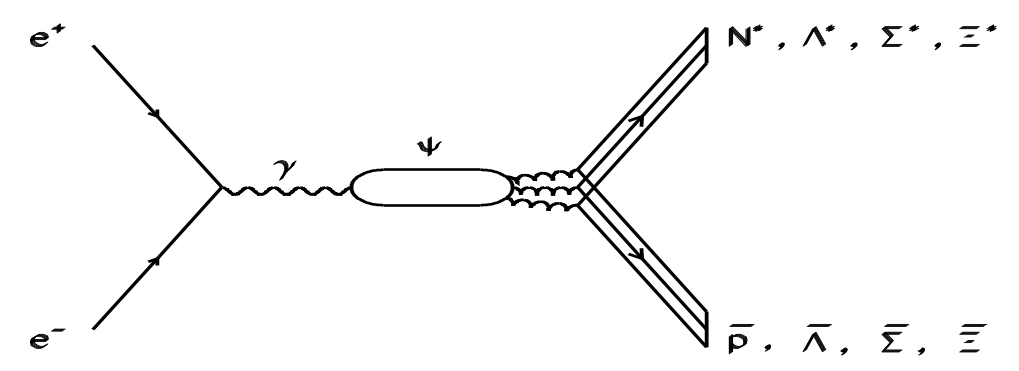

Figure 10. $\bar{p} N^{*}, \bar{\Lambda} \Lambda^{*}, \bar{\Sigma} \Sigma^{*}$ and $\bar{\Xi} \Xi^{*}$ production from $e^{+} e^{-}$collision through $\Psi$ meson.

Since the vector charmonium $\Psi$ decays through three gluons and gluons are flavor blind, the strange s quarks are produced at the same level as the non-strange $u$ and d quarks. Table 3 lists some interested $J / \Psi$ decay branching ratios [9]. The $p \bar{p}, \Lambda \bar{\Lambda}, \Sigma^{0} \bar{\Sigma}^{0}$ and $\Xi \bar{\Xi}$ are indeed produced at similar branching ratios. The branching ratios for $\bar{p} N^{*}, \bar{\Lambda} \Lambda^{*}, \bar{\Sigma} \Sigma^{*}$ and $\bar{\Xi} \Xi^{*}$ are expected to be of the same order of magnitude if one ignores the phase space effect. The $\bar{\Omega} \Omega^{*}$ channels have thresholds above or very close to the mass of $\Psi^{\prime}$ and cannot be studied here.

Table 3

$J / \Psi$ decay branching ratios $\left(\mathrm{BR} \times 10^{3}\right)$ for some interested channels [9]

\begin{tabular}{ccccccc}
\hline$p \bar{p}$ & $\Lambda \bar{\Lambda}$ & $\Sigma^{0} \bar{\Sigma}^{0}$ & $\Xi \bar{\Xi}$ & $\Lambda \bar{\Sigma}^{-} \pi^{+}$ & $p K^{-} \bar{\Lambda}$ & $p K^{-} \bar{\Sigma}^{0}$ \\
\hline $2.1 \pm 0.1$ & $1.4 \pm 0.1$ & $1.3 \pm 0.2$ & $1.8 \pm 0.4$ & $1.1 \pm 0.1$ & $0.9 \pm 0.2$ & $0.3 \pm 0.1$ \\
\hline$p \bar{n} \pi^{-}$ & $p \bar{p} \pi^{0}$ & $p \bar{p} \pi^{+} \pi^{-}$ & $p \bar{p} \eta$ & $p \bar{p} \eta^{\prime}$ & $p \bar{p} \omega$ & $K^{-} \Lambda \bar{\Xi}^{+} ?$ \\
\hline $2.0 \pm 0.1$ & $1.1 \pm 0.1$ & $6.0 \pm 0.5$ & $2.1 \pm 0.2$ & $0.9 \pm 0.4$ & $1.3 \pm 0.3$ & $K^{+} \bar{\Lambda} \Xi^{-} ?$ \\
\hline
\end{tabular}

All channels listed in Table 3 are relative easy to be reconstructed by BES. For example, for $K^{-} \Lambda \bar{\Xi}^{+}$, we can select events containing $K^{-}$and $\Lambda$ with $\Lambda \rightarrow p \pi^{-}$, then from missing mass spectrum of $K^{-} \Lambda$ we should easily identify the very narrow $\bar{\Xi}^{+}$peak. The $K^{-} \Lambda \bar{\Xi}^{+}$ channel is a very good place for studying $\Xi^{*} \rightarrow K \Lambda$. At present, not much is known about $\Xi^{*}$ resonances [9]. Only the ground $\Xi(1318)$ state and the first excitation state $\Xi^{*}(1530)$ are well established. There has not been a single new piece of data on $\Xi^{*}$ resonances since PDG's 1988 edition. Various theoretical predictions by rather different physical pictures 27,28 are not challenged due to the lack of data. With $J / \Psi$ and $\Psi^{\prime}$ experiments at BEPC and upgraded BEPCII in near future, we expect to complete the $\Xi^{*}$ resonance spectrum as well as the $N^{*}, \Lambda^{*}$ and $\Sigma^{*}$ resonance spectra. 
Among three-body channels listed in Table $3, \Lambda \bar{\Sigma}^{-} \pi^{+}$and $p K^{-} \bar{\Lambda}$ can be used to study $\Lambda^{*} \rightarrow \Sigma \pi$ and $N K ; \Lambda \bar{\Sigma}^{-} \pi^{+}$and $p K^{-} \bar{\Sigma}^{0}$ can be used to study $\Sigma^{*} \rightarrow \Lambda \pi$ and $N K$; Channels containing p can be used to study $N^{*} \rightarrow K \Lambda, K \Sigma, N \pi, N \pi \pi, N \eta, N \eta^{\prime}$ and $N \omega$. Many other channels not listed in Table 3 can also be used to study these baryon resonances.

In fact, the Feynman graph in Fig. 10 is almost identical to those describing the $N^{*}$ electro-production process if the direction of the time axis is rotated by $90^{\circ}$. The only difference is that the virtual photon here is time-like instead of space-like and couples to $N N^{*}$ through a real vector charmonium meson $\Psi$. So all $N^{*}$ decay channels which are presently under investigation at $\operatorname{CEBAF}(J L a b, \mathrm{USA}$ ) 29], ELSA(Bonn,Germany) 26], GRAAL(Grenoble, France) and Spring8(KEK, Janpan) with real photon or space-like virtual photon can also be studied at BEPC complementally with the time-like virtual photon. In addition, for $\Psi \rightarrow \bar{N} N \pi$ and $\bar{N} N \pi \pi$, the $\pi N$ and $\pi \pi N$ systems are limited to be pure isospin $1 / 2$ due to isospin conservation. This is a big advantage in studying $N^{*}$ resonances from $\Psi$ decays, compared with $\pi N$ and $\gamma N$ experiments which suffer difficulty on the isospin decomposition of $1 / 2$ and $3 / 2[30$.

Based on 7.8 million $J / \Psi$ events collected at BEPC before 1996, the events for $J / \Psi \rightarrow$ $\bar{p} p \pi^{0}$ and $\bar{p} p \eta$ have been selected and reconstructed with $\pi^{0}$ and $\eta$ detected in their $\gamma \gamma$ decay mode[31]. For selected $J / \Psi \rightarrow \bar{p} p \gamma \gamma$ events, the invariant mass spectrum of the $2 \gamma$ is shown in Fig.11. The $\pi^{0}$ and $\eta$ signals are clearly there. The $p \pi^{0}$ invariant mass spectrum for $J / \Psi \rightarrow \bar{p} p \pi^{0}$ is shown in Fig. 12 with clear peaks around 1500 and 1670 $\mathrm{MeV}$. The $p \eta$ invariant mass spectrum for $J / \Psi \rightarrow \bar{p} p \eta$ is shown in Fig. 13 with clear enhancement around the $p \eta$ threshold, peaks at 1540 and $1650 \mathrm{MeV}$; both have been determined to have $J^{P}=\frac{1}{2}^{-}$by a PWA analysis 31. From the relative branching ratio of $N^{*}(1535)$ to $\eta N$ and $\pi N[9]$, the narrow peak at $1500 \mathrm{MeV}$ in $p \pi^{0}$ mass spectrum of $J / \Psi \rightarrow \bar{p} p \pi^{0}$ is expected to be mainly due to $N^{*}(1535)$. With 23 million new $J / \Psi$ data collected by BESII in last few months, many more channels, such as $p \bar{n} \pi^{-}, \bar{p} n \pi^{+}, \bar{p} \Lambda K$, $\bar{\Lambda} K, \bar{p} p \omega, \bar{p} p \eta^{\prime}, \bar{p} p \pi^{+} \pi^{-}$, and $\bar{p} p \phi$ etc., are now under investigation.

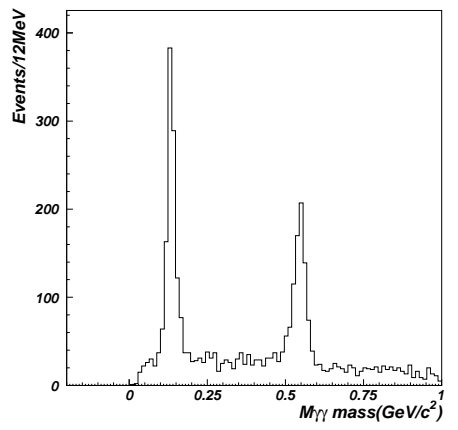

Figure 11. $\quad \gamma \gamma$ invariant mass spectrum after $4 \mathrm{C}$ fit for $J / \Psi \rightarrow \bar{p} p \gamma \gamma$

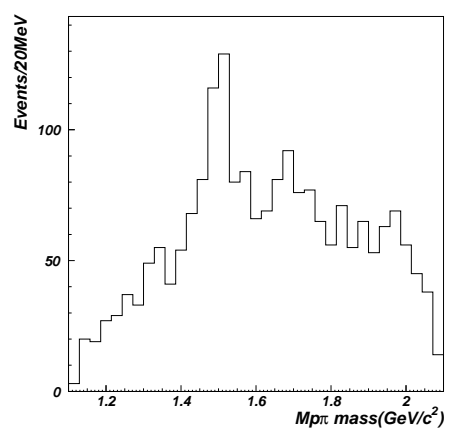

Figure 12. $\quad p \pi^{0}$ invariant mass spectrum for $J / \Psi \rightarrow$ $\bar{p} p \pi^{0}$.

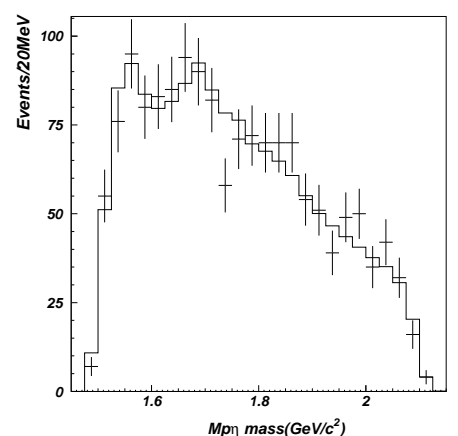

Figure 13. $\quad p \eta$ invariant mass spectrum for $J / \Psi \rightarrow \bar{p} p \eta$

On theoretical side, the coupling of $\Psi \rightarrow \bar{p} N^{*}$ provides a new way to probe the in- 
ternal quark-gluon structure of the $N^{*}$ resonances 32]. In the simple three-quark picture of baryons, as shown in Fig. 10, three quark-antiquark pairs are created independently via a symmetric three-gluon intermediate state with no extra interaction other than the recombination process in the final state to form baryons. This is quite different from the mechanism underlying the $N^{*}$ production from the $\gamma p$ process where the photon couples to only one quark and unsymmetric configuration of quarks is favored. Therefore the processes $\Psi \rightarrow \bar{p} N^{*}$ and $\gamma p \rightarrow N^{*}$ should probe different aspects of the quark distributions inside baryons. Since the $\Psi$ decay is a glue-rich process, it is also regarded as a good place for looking for hybrid $N^{*}[33$.

In summary, the $J / \Psi$ and $\Psi^{\prime}$ experiments at BEPC provide an excellent place for studying excited nucleons and hyperons $-N^{*}, \Lambda^{*}, \Sigma^{*}$ and $\Xi^{*}$ resonances. The completion of the light quark (u,d,d) baryon spectroscopy is of crucial importance for us to reveal quark gluon structure of matter.

\section{Outlook}

All PWA results summarized in this paper are based on 7.8 million $J / \Psi$ events collected by the old version of the BES detector (BESI) before 1996. Since November 1999, 23 million new $J / \Psi$ events have been collected with the new improved BES detector (BESII) and 27 million more $J / \Psi$ events are going to be taken before next May.

A major upgrading of the collider to the BEPC2 has been approved by the Chinese central government very recently. A further one order of magnitude more statistics is expected to be achieved. Such statistics will enable us to perform partial wave analyses of plenty important channels for both meson spectroscopy and baryon spectroscopy from the $J / \Psi$ and $\Psi^{\prime}$ decays. We expect BEPC2 to play a very important unique role in many aspects of light hadron spectroscopy, such as hunting for the glueballs and hybrids, extracting $u \bar{u}+d \bar{d}$ and $s \bar{s}$ components of mesons, and studying excited nucleons and hyperons, i.e., $N^{*}, \Lambda^{*}, \Sigma^{*}$ and $\Xi^{*}$ resonances.

Acknowledgements: It is a pleasure to thank Prof. Martin Faessler and his staff for the successful organization of LEAP2000 on the nice Venice island and the invitation. Deep thanks to my collaborators in the BES experiment at BEPC.

\section{REFERENCES}

1. BES Collaboration, J.Z.Bai et al., Nucl. Indtr. Methods A 344 (1994) 319.

2. L.Köpke and N.Wermes, Phys. Rep. 174 (1989) 67.

3. BES Collaboration, J.Z.Bai et al., Phys. Lett. B472 (2000) 207.

4. BES Collaboration, J.Z.Bai et al., Phys. Lett. B472 (2000) 200.

5. BES Collaboration, J.Z.Bai et al., Phys. Lett. B446 (1999) 356.

6. BES Collaboration, J.Z.Bai et al., Phys. Lett. B476 (2000) 25; Phys. Lett. B440 (1998) 217.

7. Z.J.Guo, IHEP Ph.D thesis (2000); BES Collaboration, to be published.

8. BES Collaboration, X.Y.Shen, talk given at 4th Int. Conf. on Hyperons, Charm and Beauty Hadrons, Valencia, Spain, June 2000. Submitted to Nucl. Phys. Proc. Suppl.

9. Particle Data Group, Euro. Phys. J. C15 (2000) 1. 
10. D.V.Bugg, I.Scott, B.S.Zou et al., Phys. Lett. B353 (1995) 378.

11. D.V.Bugg, L.Y.Dong and B.S.Zou, Phys. Lett. B458 (1999) 511.

12. J.Chen, X.Q.Li and B.S.Zou, Chin. Phys. Lett. (2000) in press.

13. J.Chen, X.Q.Li and B.S.Zou, Phys. Rev. D62 (2000) 034011.

14. D.V.Bugg, M.Peardon and B.S.Zou, Phys. Lett. B486 (2000) 49.

15. A.Kirk, Phys. Lett. B489 (2000) 29; and references therein.

16. A.V.Anisovich et al., Phys. Lett. B449 (1999) 145.

17. S.Braccini, Nucl. Phys. A655 (1999) 143c.

18. DM2 Collaboration, A.Falvard et al., Phys. Rev. D38 (1988) 2706.

19. L.Li, B.S.Zou and G.L.Li, Preprint hep-ph/0010196.

20. A.V.Anisovich, A.V.Sarantsev and V.A.Anisovich, Phys. Lett. B395 (1997) 123.

21. C.Amsler and F.E.Close, Phys. Rev. D53 (1996) 295; D.Weingarten, Nucl. Phys. B (Proc. Suppl.) 53 (1997) 232.

22. P.R.Page et al., Phys. Rev. D59 (1999) 034016.

23. BES Collaboration, G.F.Xu et al., Nucl. Phys. A675 (2000) 337c.

24. BES Collaboration, Y.C.Zhu et al., Proc. of Workshop on the Developing Strategy of HEP in China, p.17.

25. N.Isgur, Baryon'98, p.1, ed. D.Menze and B.Metsch, World Scientific, 1999.

26. E.Klempt, ibid, p.25.

27. S.Capstick and N.Isgur, Phys. Rev. D 34 (1986) 2809.

28. L.Glozman, W.Plessas, K.Varga and R.Wagenbrunn, Phys. Rev. D 58 (1998) 094030.

29. V.D.Burkert, Nucl. Phys. A 623 (1997) 59c.

30. R.Workman, Few Body Syst. Suppl. 11 (1999) 94.

31. BES Collaboration, H.B.Li et al., Nucl. Phys. A675 (2000) 189c; B.S.Zou et al., hep$\mathrm{ph} / 0004220$.

32. B.S.Zou et al., hep-ph/9909204; C.Carimalo, Int. J. Mod. Phys. A2 (1987) 249.

33. S.Capstick and P.Page, Phys. Rev. D 60 (1999) 111501. 